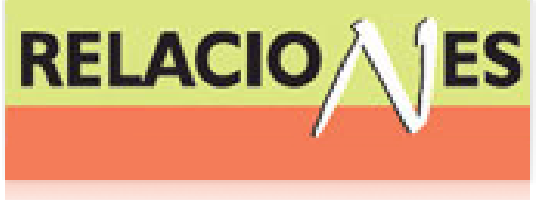

Relaciones. Estudios de historia y sociedad ISSN: 0185-3929

relacion@colmich.edu.mx

El Colegio de Michoacán, A.C

México

Vázquez Parra, José Carlos

El vínculo y la racionalidad, tres enfoques de la noción ética del cuidado del otro. Una aproximación desde la teoría de la racionalidad elsteriana

Relaciones. Estudios de historia y sociedad, vol. XXXIV, núm. 134, 2013, pp. 349-364

El Colegio de Michoacán, A.C

Zamora, México

Disponible en: http://www.redalyc.org/articulo.oa?id=13726972011

- Cómo citar el artículo

Número completo

- Más información del artículo

- Página de la revista en redalyc.org

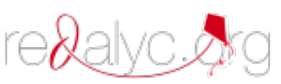

Sistema de Información Científica

Red de Revistas Científicas de América Latina, el Caribe, España y Portugal

Proyecto académico sin fines de lucro, desarrollado bajo la iniciativa de acceso abierto 


\title{
El vínculo y la racionalidad, tres enfoques de la noción ética del cuidado del otro. Una aproximación desde la teoría de la racionalidad elsteriana
}

\author{
José Carlos Vázquez Parra* \\ INSTITUTO TECNOLÓGICO Y DE ESTUDIOS SUPERIORES DE MONTERREY, CAMPUS \\ MONTERREY
}

En la actualidad el cuidado del otro es un tema poco considerado por la mayor parte de la población, pareciendo que en el espíritu de la postmodernidad el hombre tendiera a un cuidado plena y únicamente de sí mismo. Sólo a partir de la adopción de estados alternativos y basados en nociones éticas, se podrá revertir tal tendencia, aunque para ello, se requiere una reflexión de los constructos de la relación yo-otro.

(Equidad, responsividad, elección, racional, ética)

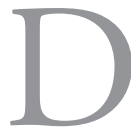

espués de la segunda guerra mundial, el hombre consideró que ya se había presenciado el peor atentado a lo que significa ser humano, sin embargo, parece ser que entre más avanzan los años, se busca dejar esta aseveración atrás, encontrando nuevos niveles de deshumanización y despersonalización. La discriminación y el maltrato que en los últimos años se ha potencializado hacia los trabajadores, los pobres, las mujeres, los indígenas, entre otros grupos minoritarios, muestra un presente desafiante y un futuro en que parece ser que todo es más importante que la equidad, el cuidado del otro y la responsabilidad que se le debe a éste por el solo hecho de ser humano.

Como respuesta hacia este reto deshumanizante, es que no únicamente se han retomado en las mesas de diálogo, los clasicos postulados filosóficos y sociales que respaldan el cuidado del otro, como

*Jvazquez24hrs@gmail.com 
serían las aportaciones de Emmanuel Lévinas o inclusive de John Rawls, sino que también se han gestado nuevos enfoques, que a partir de análisis interdisciplinarios de conceptos y nociones éticas aportan una nueva visión sobre el significado y la importancia que se tiene al hablar y cuidar del otro.

Uno de estos nuevos enfoques es el propuesto por Jon Elster, filósofo y teórico social noruego, quien considera que las actuaciones en beneficio de los otros, es decir, el cuidado y actos que buscan el bienestar del otro sobre el de uno mismo, juegan un papel fundamental en el entendimiento de las motivaciones de la actuación humana en general.

Elster ha dedicado gran parte de su vida y obra al análisis de los diferentes factores que limitan y condicionan la racionalidad de los actos, buscando explicar los elementos necesarios para alcanzar actuaciones consideradas lo más racionalmente posibles. En dicho proceso, Elster reconoce que aunque el acto para ser racional debe buscar ser la mejor opción para alcanzar los fines del individuo con base en sus creencias y sus deseos, hay casos en que los fines ajenos y el beneficio del otro, aun siendo contrario a las preferencias y fines del actor, puede llevar a actuaciones plenamente racionales. Para este filósofo noruego, el cuidado del otro es una ocasión muy favorecedora para analizar y estudiar nuevas nociones motivadoras de la actuación racional humana.

Para los fines del presente escrito, se ha decidido hacer un abordaje introductorio a partir de dos propuestas teóricas, que aunque parecen ser tan distintas, encuentran un punto indirecto de concurrencia en la teoría y en algunas de las aportaciones de un tercer enfoque. Los postulados de los autores introductorios son aquellos que se considera permiten encontrar una intersección y un espacio reflexivo de las propuestas que Jon Elster ofrece sobre las actuaciones que se hacen buscando el beneficio y el cuidado del otro.

Primeramente, se presenta el enfoque de Emmanuel Lévinas, a quien a partir del análisis de algunas de sus obras se pretende señalar de una manera sencilla, especificando la esencial importancia que este autor pone en la relación yo-otro así como a la responsividad nacida de tal correspondencia. Por otro lado, se exponen algunos de 
los planteamientos que con referencia al tema se consideran más determinantes de la obra de John Rawls, enfocándose primordialmente en su ideal de justicia como equidad y en su segundo principio de justicia, es decir, su postulado sobre la desigualdad.

Cabe aclarar que con este artículo no se pretende agotar el amplio marco teórico que engloba la rica temática del cuidado del otro, ya que tanto el abordaje desde la visión de Lévinas como desde la postura de Rawls bien ameritaría un escrito individual para cada uno de ellos. Sin embargo, lo que si se busca, es presentar un enfoque diferente, que girando en torno a nociones de racionalidad y elección, permita plasmar un nuevo panorama de análisis sobre este elaborado e importante tema, teniendo como sustento teórico las propuestas conceptuales de Jon Elster.

\section{LA ASIMETRÍA COMO BASE DE LA RELACIÓN YO-OTRO}

Emmanuel Lévinas, filósofo judío nacido en Lituania, es sin duda uno de los mayores exponentes de la necesidad filosófica de volverse al otro, es decir, de negar la primacía ontológica, defender la alteridad y dar al otro el lugar prioritario de la relación yo-otro. Este último punto (la relación yo-otro) se vuelve toral en la propuesta de Lévinas, en la que el vínculo relacional es el punto central de cualquier análisis de reciprocidad o no reciprocidad de la actuación humana.

Es común encontrar que la teoría levinasiana tienda a ligarse a otras propuestas como las de Martin Buber y Franz Rosenzweig por ser todos ellos, filósofos que se apartan de la idea tradicional de filosofía del ser, rescatando la emocionalidad humana y retomando la importancia del sujeto sobre el saber. Según Patiño (2010) tales filósofos pueden ser incluso llamados filósofos del diálogo, haciendo alusión al sentido de que, desde su perspectiva, el entendimiento del logos se hace en razón de dos, es decir, en razón de una relación con el otro. Para esta estudiosa de la ética, lo ético en Lévinas se encuentra en el yo-tú del diálogo, en la inmediatez de la relación con el otro (p. 46).

Esta relación yo-otro, según lo expone el propio Lévinas en su obra Totalidad e infinito (2006), está basada en un trato asimétrico, es decir, que presenta una imposibilidad moral de exigir al otro lo 
que tiende a exigirse a sí mismo, ya que éste (el otro) se encuentra en una posición privilegiada, imponiendo sus intereses a los del yo.

Este señalamiento (de la relación asimétrica yo-otro) puede también ser apreciado en la obra Humanismo del otro hombre (2006), en que Lévinas expone que ser hombre significa ser para otro. En cuanto este sentido, Patiño (2010) agrega, que tal noción de hombre lleva a un desplazamiento del yo soberano, común en la filosofía tradicional, para dar paso a un nuevo yo rehén de el otro, a partir de una relación que no es otra que la relación ética nacida de la mirada vuelta hacia el rostro del otro.

Lévinas pondrá especial atención en esta nueva concepción humanista, por medio de la cual se busca salvaguardar la preocupación por el otro, por su propiedad, por su dignidad y por sus intereses. Es tal la importancia de ver por otro que en Ética e infinito (2008) Lévinas llega a aseverar que ante las necesidades del otro no cabe lugar para la indiferencia del yo, ya que sólo a partir de éste (de la atención y procuración del bienestar del otro) es que se puede llegar a descubrir el bien. Para la propuesta levinasiana, el cuidado del otro se basa plenamente en la relación interpersonal, en la cual la atención por el otro no se considera una opción, sino una obligación que el yo no puede evitar.

\section{LA JUSTICIA ECUÁNIME HACIA EL OTRO}

En un sentido muy diferente a lo planteado con el abordaje de Lévinas, antes de poder hacer lo propio con John Rawls es necesario señalar un factor que es interesante considerar dentro de su noción de justicia como equidad. En 1985, Rawls publicó su ensayo Justice as Fairness: Political not Metaphysical, haciendo una revaloración de la noción de justicia, llevándola desde su noción griega de "Constante y perpetua voluntad de dar a cada uno su derecho", a una postura mucho más social, en la que la justicia tiende a la búsqueda de una igualdad o equilibrio entre los individuos intervinientes. Algo que llama la atención de su idea es que ésta no sólo se percibe como concepto, sino como una actitud de justicia, como un estado o ánimo para actuar justamente. 
Sin embargo, el principal problema que se presenta en la propuesta de Rawls, y que es necesario explicitar antes de profundizar en su noción, es el llegar a entender el espíritu del concepto, es decir, la esencia que este giro conceptual propone, ya que el simple hecho de traducir la idea central de su ensayo, lleva a generar diferentes concepciones de la misma, siendo algunos de estos planteamientos muy alejados de la pretensión rawlsiana.

La noción de John Rawls de un concepto de justicia como estado, tiene plena relación con el uso del término fairness, el cual es más complejo de explicar que simplemente el equivalente de equidad, que es la traducción que se propone en la mayoría de las ocasiones. Fairness es una palabra que comúnmente es traducida a partir de un sinónimo, ya que el sentido objetivo de la palabra no existe en nuestra lengua, es decir, fair denota a justo, por lo que fairness debería ser algo así como "la justedad", concepto que generaría una redundancia al llevar a una traducción de "justicia como justedad" y que por ello se deriva a la idea de equidad. Sin embargo, independientemente de lo anterior, Justice as fairness, no sólo apunta a una característica o noción generada a partir del concepto de justicia como equidad, sino que engloba un carácter y un estado de ánimo que se tiene a partir del ejercicio de tal noción de justicia.

Tal situación genera la necesidad de considerar que aunque equidad sea una palabra que se contempla análoga a la idea de fairness, igualmente podrían usarse otros sinónimos como ecuanimidad, imparcialidad, equilibrio e inclusive igualdad, los cuales pueden ser esclarecedores de la postura rawlsiana. Sin embargo, de entre todas estas opciones, es ecuanimidad, uno de los conceptos al que se debe poner especial atención, ya que con base en la Real Academia de la Lengua Española (2001), se puede definir como "igualdad y constancia de ánimo", lo cual, más que ser una situación, denota una actitud, un estado mental, mismo caso que es buscado por Rawls con el término fairness y del cual se puede enlazar consecuentemente la noción de justicia.

Así, es que para entender el sentido original de Justice as Fairness se debe considerar el cambio de "justicia como equidad" a "justicia como ecuanimidad", ya que ésta es una traducción más precisa del 
sentido que Rawls propone y que, además, permite esclarecer los diferentes constructos que se han generado respecto a la noción de justicia en la época actual.

Una vez aclarado este punto se puede profundizar en el papel que tiene la justicia como ecuanimidad en el sentido que tiene Rawls del cuidado del otro, cuestión, que desde su tradición deontológica, es abordada de una forma muy diferente a lo que se analizó con Lévinas. Para Rawls, la noción de justicia tiende a suponerse a partir de la observación de la norma, y el cuidado del otro se prevé como parte de tal ideal o estado de justicia en el cual se desarrolla la sociedad y no tanto como resultado de una relación. Sin embargo, para que tal ecuanimidad pueda darse y se alcance la armonía social, el filósofo expone que se deben respetar los principios pactados políticamente, ya que éstos se han generado con la pretensión y la finalidad de beneficiar mayormente a todos por igual. De esta manera es que la concepción de justicia como ecuanimidad rawlsiana está muy delimitada del ámbito de la relación personal, es en la norma y no en la relación que se entiende el sentido de justicia.

En su obra Liberalismo politico (2006), John Rawls contempla que su noción de justicia como ecuanimidad debe ser llevada al ámbito de lo político, considerando que la concepción que se tiene de persona debe dejar atrás al individualismo y enfocarse de mayor manera en un aspecto social. Profundizando en dicha propuesta, este filósofo estadounidense expone que toda sociedad bien ordenada es aquella en que los ciudadanos aceptan de manera común los principios de justicia.

En su obra magna, Teoría de la justicia (1997), Rawls presenta y desarrolla una serie de principios que buscan hacer que todos los individuos formen parte del ideal de justicia como ecuanimidad, buscando primordialmente que cada persona tenga un derecho igual al esquema de libertades básicas que tienen el resto de los ciudadanos. Sin embargo, tales principios también contemplan consideraciones especiales ante las desigualdades que pudieran presentarse, precisando que éstas sólo deberán considerarse cuando resulten en un mayor beneficio para los menos aventajados de la comunidad (principio de diferencia), es decir, sólo se da la desigual- 
dad si ésta es a favor de los menos favorecidos, previendo siempre, la justa igualdad de oportunidades.

Es en el segundo de sus principios, en que Rawls estima que aunque exista un factor de desigualdad, se sigue siendo justo, pues tal situación ha sido pactada y fijada por todos los miembros de la sociedad, considerando que es lo que mayormente beneficia a todos. Tal idea de cooperación grupal es lo que nos acerca a la noción de cuidado del otro, aunque en este caso se vuelve en una atención comunitaria y no tanto en una relación yo-otro. Para Rawls, la sociedad es un sistema justo de cooperación que se sigue a partir de lineamientos pactados y fijados por sus miembros, considerando que esto es lo que mayormente beneficia a todos.

La cooperación entre los individuos, según Rawls, debe guiarse a partir de reglas públicamente reconocidas, así como por procedimientos aceptados por cada uno de los cooperadores ya que la cooperación debe implicar la idea de condiciones justas y ecuánimes. Para lograr tal objetivo, se deben considerar primero los dos famosos ejercicios mentales que Rawls propuso como requerimientos para garantizar la justa igualdad de oportunidades; la posición original y el velo de la ignorancia. Sólo a partir de haber ejercido tales nociones es que cualquier idea de reciprocidad, dentro de la concepción rawlsiana, se dará entre ciudadanos libres e iguales y así como en un entorno considerado justo.

Para el sentido rawlsiano, el cuidado del otro se da a partir de que todos los ciudadanos gocen de libertad en la medida que se conciben a sí mismos y unos a otros como poseedores de una capacidad moral para tener una concepción de bien. El trasfondo justo de este mundo social lo da la concepción política, por lo que es la razón pública aquella en la que los ciudadanos pueden entender su papel dentro de la misma, hacia los otros como hacia sí mismos. La persona razonable no es considerada aquella que sea altruista, ya que es a partir de los fines racionales propios que se llegan a proponer términos equitativos, que siendo aceptados por todos, pueden generar un beneficio de una manera más equitativa.

La aplicación de la justicia no sólo es dentro de una actuación específica, es decir, en la relación yo-otro, sino que se vuelve parte 
del actuar constante de la persona a partir del ambiente donde se desenvuelve. La ecuanimidad, como base del constructo de la noción de justicia propuesta por Rawls, llega a ser un estado pleno de actuación del individuo en relación con el otro, ya que aunque en un inicio es particularmente usado para la formulación de normas o en la creación de mismo Estado, lleva a que una vez que el individuo lo alcanza, pueda ser aplicado al resto de sus actos.

En contraposición a Lévinas, para Rawls la relación yo-otro toma un sentido político en el que sobresale la relación yo-otros, esperando que así como el individuo ve por los demás, esos otros vean por él. Sólo de esta manera se alcanza el estado de justicia como ecuanimidad que da paso a la sociedad armónica rawlsiana.

\section{LA RELACIÓN YO-OTRO COMO CAMINO A LA RACIONALIDAD}

De forma alterna a los anteriores enfoques, que se basaban en el vínculo individual o político del yo-otro, Jon Elster nos propone un análisis un tanto alternativo, en el cual la relación entre los individuos no solamente responde a cuestiones mucho más instrumentales, es decir, apegadas a un deber o a un interés individual, sino que incluso, el cuidado del otro se convierte en un elemento importante en la búsqueda de la racionalidad de los actos.

Concentrándose en la cuestión de la racionalidad como característica formal de las acciones individuales y siguiendo una terminología propuesta por Rawls en su obra Teoría de la justicia, Jon Elster aborda por primera ocasión, en su libro Uvas amargas $(1988,9)$, una concepción propia de lo que entiende por racionalidad, diferenciando entre lo que él llamaría la teoría estricta, es decir, los planteamientos que se había hecho anteriormente y su propia propuesta, la teoría amplia de la racionalidad individual.

Elster $(1988,9)$ explica que la teoría existente de la racionalidad pone todo su énfasis en la consistencia de las razones del acto, dejando sin examinar aspectos como la capacidad de juicio en la recopilación de información o la autonomía en la generación de deseos y preferencias. Así, este autor considera que aunque la explicación de la racionalidad existente no es incorrecta, es demasiado estricta y por 
ello una gran variedad de actuaciones humanas, como el altruismo o el cuidado del otro pueden llegar ser confusas en el momento de valorarse como racionales.

Uniéndose a la noción de Donald Davidson, de que toda acción para considerarse racional debe estar relacionada con las creencias y los deseos del agente, Elster $(1988,13)$ manifiesta que la valoración de la racionalidad de un acto debe poner especial atención en la fundamentación racional de sus razones, ya que más allá de la consistencia de tales elementos, se debe garantizar que éstos son a su vez racionales.

Elster $(2010,69-70)$ expresa que la forma ideal de explicar una acción consiste en demostrar que es racional; sin embargo, para que este señalamiento sea adecuado, se tiene que demostrar que se tienen las creencias que sustenten el acto en sí, así como el deseo o preferencia de hacerlo, considerando que para ello, la acción deberá ser óptima a la luz de la totalidad de sus razones. La racionalidad elsteriana $(1988,29)$, si se puede llamar de ese modo, es aquella que permite estudiar detenidamente la naturaleza sustancial de los deseos y las creencias involucradas en los actos humanos, mismos que pueden responder para sí, como para el otro.

Una creencia, conforme a la propuesta de Elster, se considera racional a partir de la relación que tiene ésta con la evidencia que se posee, considerando la concordancia de tal información y la historia causal de la misma. Con base en esta importante relación de la evidencia con las creencias, es que Elster $(1988,30)$ prevé que el nivel óptimo de evidencia es un problema toral que debe ser considerado al hablar de racionalidad, ya que no es fácilmente definible y tanto la falta como el exceso de información puede ser altamente perjudiciales en la generación de la creencia. Por ello, el autor ha expresado que la caracterización positiva de las creencias racionales es la noción de juicio, ya que ésta permite que el agente sintetice la información vasta y difusa que puede llegar a tener sobre una idea, evitando que le dé una importancia o peso indebido a información irrelevante que puede llegar a afectar la racionalidad.

En cuanto a los deseos o preferencias, como segundo aspecto por considerar dentro de la Teoría Amplia de la Racionalidad, el autor 
estima racionales a aquellos deseos que han sido formados correctamente, es decir, que no han sido distorsionados por procesos causales irrelevantes y conservan su autonomía. Elster (1988, 36-37) expresa que así como el juicio es para las creencias racionales, la autonomía es la caracterización positiva de las preferencias, considerando que un deseo autónomo es aquel que ha sido escogido, adquirido o modificado deliberadamente, ya sea por un acto de voluntad o por un proceso de planificación del carácter, es decir, que surge como resultado de la educación o rasgos de la personalidad propios del individuo.

De esta manera, los actos, desde la perspectiva elsteriana, deben llevar consigo un análisis mucho más profundo respecto a sus motivaciones o razones, siendo no la excepción, aquellas actuaciones que se hacen directa o indirectamente en beneficio o en cuidado del otro.

Para Elster $(1989,50)$, la idea de un estado de naturaleza en el cual las personas viven en el presente y sólo se preocupan por sí mismas, pensamiento muy común en Hobbes, es altamente cuestionable. Para este filósofo noruego, las personas tienden a hacer comparaciones constantes acerca de lo que es valioso para ellas, considerando no solo la valía, sino también los deseos, las creencias y por supuesto, el tiempo.

En su obra Tuercas y tornillos: Una introducción a los conceptos básicos de las ciencias sociales (1989, 58-60), Elster se cuestiona sobre tal espíritu individualista, aunque por otro lado, es puntual acerca de las opciones de instrumentalización e interés a largo plazo que pueden ser motivos de las actuaciones que se consideran altruistas. Quizá, expresa este autor, las acciones tendientes a la ayuda y cuidado del otro (altruistas) deriven en realidad del interés propio, ya sea por el prestigio que esto genera socialmente o por el interés de una reciprocidad posterior, sin embargo, siempre se tiene la opción de actuar egoístamente.

Para Jon Elster, el cuidado del otro se ve configurado a partir de la instrumentalización de la conducta, es decir, el elegir un acto en bienestar del otro responde a fines específicos, que si bien pueden ser imperceptibles a corto plazo, tienden a objetivos precisos poste- 
riores. Los deseos de satisfacción inmediata de preferir actuar para sí y no para otros, se ven subordinados en la escala de preferencias de los deseos a partir de la optimalidad de la evidencia con que se cuenta, misma que responde a las creencias sociales, culturales e incluso morales del cuidado del otro.

Elster reconoce, sin embargo, que existen factores emocionales que pueden alterar la elección de un acto altruista, mismos que motivados por deseos de inmediata satisfacción, pueden llevar a que el individuo caiga en una miopía temporal, es decir, no ver más allá de su temporalidad próxima. Esta situación (miopía temporal) es aquella en que la persona es arrastrada a una urgencia de satisfacción individual que afecta a la recabación de información, sesgando la calidad de la evidencia y ocasionando creencias erróneas, oscuras y finalmente irracionales. Además, la motivación enteramente generada a partir de tales emociones ponen en entredicho la autonomía misma del deseo y de las preferencias, afectando a su vez su racionalidad.

De esta manera, la actuación que se da para la satisfacción plenamente individual se convierte en una elección que bien pudiera cuestionarse como irracional, ya que aun siendo beneficiosa para la persona, puede ser contraria a una escala de preferencias de mayor temporalidad. El cuidado del otro se convierte, bajo el parámetro de la teoría elsteriana, en una actuación que respeta o por lo menos, considera los bienes futuros, lo cual permite tanto deseos más autónomos, como creencias sustentadas en un mejor juicio.

La teoría de la racionalidad elsteriana, encuentra en el cuidado del otro, un ejemplo preciso de la importancia del análisis de las motivaciones del acto, mismo que debe ir más allá de la evaluación de consistencia y hacer hincapié en la racionalidad plena de la línea causal. Elster, de manera distinta a los anteriores autores (Lévinas y Rawls) más que sustentarse en la calidad del vínculo o en la reciprocidad de la relación yo-otro, se basa en el sustento racional de dicho enlace, dando pautas que permiten al yo, explorar, entender o generar nuevos vínculos eficaces, eficientes y racionalmente beneficiosos con el otro que le rodea. 


\section{Puntos de Encuentro}

El cuidado del otro es un tema que, como se había mencionado en un inicio, parece tomar mayor importancia cada día y, sin embargo, trae consigo tantas perspectivas de abordaje que su interpretación y análisis suele darse de manera confusa. Los tres planteamientos, anteriormente explicados, ofrecen perspectivas muy propias de atención ante este tipo de actuaciones (actos que tienden al beneficio y cuidado del otro), ya que según los constructos de cada una de las nociones, se puede determinar la forma en que se da tal acto, visto desde una postura de responsividad, desde un objetivo de justicia o, bien, desde una valoración de racionalidad.

El estado de responsividad, como ya se había mencionado, genera una obligación directa sobre las necesidades del otro, es decir, el enlace yo-otro es plenamente asimétrico y es más importante la conservación y cuidado del vínculo que cualquier otro principio, incluso colocándose sobre la noción que cotidianamente tenemos de justicia.

El otro, visto desde el estado ecuánime, requiere ser tratado con equidad, es decir, se le cuida tanto como se quisiera que se cuidara de uno, pero no sólo en un acto, sino en todo momento de la relación. Sin embargo, desde este enfoque, las necesidades del yo-otro siempre deben tender a lo planteado según los principios de justicia pactados políticamente, respetando el ejercicio mental de la posición original y el velo de la ignorancia.

En el caso del calificativo racional, se da una situación un tanto más compleja, ya que se requiere de una reflexión profunda de las creencias y los deseos que tiene el individuo sobre su lugar en la relación con los otros. El ideal de esto, sería la aplicación de nociones éticas como base de las creencias, ya que ello implicaría una reflexión fundada en constructos que cuentan con evidencias lo suficientemente óptimas para evitar futuros cuestionamientos sobre la capacidad de juicio aplicada.

El fomento de la utilización de postulados éticos para la toma de decisiones en nuestro actuar hacia los otros, no sólo nos beneficia en la valoración de racionalidad de los mismos, ya que la mayoría de los 
principios éticos traen consigo una fuerte reflexión moral, sino que además, nos permiten que la relación yo-otro trascienda a un nivel superior, es decir, a una correspondencia ética.

El enfoque elsteriano, a diferencia de los anteriores, respeta la capacidad reflexiva del individuo, así como su papel activo en la decisión de actuar para sí o para otros, ya que como siempre lo ha mencionado Jon Elster, la racionalidad de los actos no es la norma de todas nuestras acciones. Además, el cuidado del otro, planteado como camino para alcanzar la racionalidad, no sólo subsana las restricciones de información que se presentan bajo el velo de la ignorancia rawlsiano, sino que también, recupera el principio de justicia y ecuanimidad que se ve muy difuminado en la propuesta levinasiana.

\section{ÚlTIMAS CONSIDERACIONES}

El espíritu de la postmodernidad alaba las diferencias humanas, sin embargo, tiende hacia la estandarización y a la búsqueda de instituir concepciones básicas que logren regular a todos bajo las mismas nociones. Tal pretensión de alcanzar un estado de ecuanimidad entre los individuos se vuelve cada día en una actitud más difícil de lograr, pues nuevas aportaciones como las del libertario Robert Nozick (1988) hacen demasiado hincapié en la necesidad del respeto al individualismo. Algo semejante le sucede a la propuesta levinasiana, ya que su planteamiento de asimetría en la relación yo-otro, además de parecer algo utópico en la sociedad moderna, suele llegar a apreciarse como algo altruistamente nocivo en que se violentan los derechos propios del individuo a cambio del respeto y satisfacción de la relación con los otros.

La propuesta de racionalidad que se alcanza a partir de la optimalidad de las creencias del individuo, en contraposición a los anteriores enfoques, permite que la relación yo-otro sea llevada a un ámbito más reflexivo y cotidiano, respetando las diferencias y deseos de cada ente de la relación yo-otro. Además, el papel que tiene el individuo al ser generador de sus propias decisiones le permite tener un lugar mucho más activo dentro de la relación con los demás, siendo él mismo quien elija actuar para sí o para otros. 
Los planteamientos de ecuanimidad, responsividad o racionalidad, son algunos de los ánimos o conjunto de actitudes, tanto externas como internas, que se pueden llegar a tener en torno a una situación, que en este caso sería la relación yo-otro. A su vez, son propuestas generadoras de espacios donde se construyen los distintos constructos que giran en torno de la noción ética del cuidado del otro, llegando a ser influencia, condición, guía e incluso, determinantes de las decisiones, que respecto al mismo tema tomen los individuos.

\section{Conclusiones}

La relación con el otro y el cuidado del mismo, es uno de los puntos que más ha ganado importancia en las últimas décadas en que la tecnología, el ritmo acelerado de vida y la falta de encuentros con otros individuos nos lleva, inevitablemente, a un quiebre del humanismo y la entrada de una tendencia despersonalizante cada vez más común.

Las aportaciones de Rawls y Lévinas a este tema, aunque tienen claras diferencias, gozan de un punto común, la importancia que ambos le dan a la relación, es decir, al vínculo yo-otro. Sin embargo, ninguno de los dos enfoques logra salir airoso desde una reflexión ética, ya que mientras que a Rawls se le critica por su ejercicio mental con información restringida (el velo de la ignorancia), a Lévinas se le reprocha la falta de consideración del principio de justicia que pueden darse en sus relaciones asimétricas.

De esta manera, no siendo contraria, sino complementaria, es que la propuesta de Jon Elster se convierte en un nuevo camino para el entendimiento de tal relación yo-otro. El enfoque de este filósofo noruego de voltear nuestra mirada a los procesos de racionalidad implícitos en el comportamiento altruista, permiten entender no sólo el grado instrumentalizante de la relación con los otros entes que nos rodean, sino también la falta de racionalidad que se tendría de no actuar a favor de ellos. La búsqueda de enlaces que respeten la racionalidad de la actuación humana nos acerca un poco más al entendimiento del ideal ético que se incluye en el cuidado del otro, ya que, como se había mencionado, el manejo de las 
nociones éticas como fundamento de las creencias y los deseos, no sólo le da consistencia al actuar, sino también una valoración de racionalidad.

De esta manera, el vínculo y la resultante actuación del cuidado del otro, no sólo debe fundamentarse en un pacto político y sus normas resultantes, ni tampoco puede quedarse en una relación de entrega plena del yo al otro, sino que a partir de la reflexión ética, la relación yo-otro debe apreciarse como un camino de análisis ético y de racionalidad en el comportamiento humano tanto para sí como para sus semejantes.

\section{BIBLIOGRAFÍA}

Campbell, Tom, La Justicia: Los principales debates contemporaneos, Barcelona, Gedisa, 2002.

Elster, Jon, Uvas amargas. Sobre la subversión de la racionalidad, Barcelona, Península, 1988. , Tuercas y tornillos, Barcelona, Gedisa, 1989. , Sobre las pasiones. Emoción, adicción y conducta humana, Barcelona, Paidós, 2001. , Alquimias de la mente: Racionalidad y emociones, Barcelona, Paidós, 2002. , La explicación del comportamiento social: más tuercas y tornillos para las ciencias sociales, Barcelona, Gedisa, 2010.

LÉvinas, Emmanuel, Humanismo del otro hombre, México, Siglo XXI, 2006.

, Totalidad e infinito: Ensayo sobre la exterioridad, Salamanca, Sígueme, 2006. Etica e infinito, Madrid, A. Manchado, 2008.

Nozick, Robert, Anarquía, estado y utopia, México, Fondo de Cultura Económica, 1988.

Patiño, Susana, La responsividad ética, Monterrey, Plaza y Valdes Editores, 2010.

RAE, Diccionario de la Real Academia Española, R. A. Española, Ed., Recuperado el 15 de Marzo de 2011, de http://www.rae.es/ rae.html 
Rawls, John, "Justice as Fairness: Political not Metaphysical”, Philosophical Review , 1985, 164-194.

, Teoría de la justicia, México, Fondo de Cultura Económica, 1997.

, Liberalismo político, México, Fondo de Cultura Económica, 2006.

FECHA DE RECEPCiÓn DEL ARTÍCUlO: 12 de abril de 2011

FECHA DE RECEPCIÓN DE LA VERSIÓN FINAL: 31 de octubre de 2011 